\title{
Impact of AIDS on neonatal care
}

The emergence of HIV infection has forced health professionals to review their routine clinical practices. In the United Kingdom this has only recently become a problem in obstetrics and neonatal paediatrics as most HIV infection has been in adult men. Of the 8794 people identified as HIV antibody positive up to the end of June 1988 , only $889(10 \%)$ were female. ${ }^{1}$ Most of these were intravenous drug abusers $(54 \%), 25 \%$ acquired the infection through heterosexual contact and a much smaller number (7\%) had been infected by transfusion of blood or its components. Clinical management of both the mother with proved HIV infection, or who is at high risk of infection, and her baby has become a matter of concern for all maternity departments as the number of infected women is increasing and experience from the United States suggests this trend will continue. In Massachusetts, screening all newborn infants early in 1987 showed that $2 / 1000$ women giving birth must have been positive for HIV antibody, with a seropositive rate of $8 / 1000$ in inner city hospitals. ${ }^{2}$

The Royal College of Obstetricians and Gynaecologists (RCOG) has published a report in conjunction with the British Paediatric Association and the Royal College of Midwives on 'Problems Associated with AIDS in relation to Obstetrics and Gynaecology', which also includes the care of the newborn infant. $^{3}$ The aim of the report is to produce guidelines for clinical practice. Many of the precautions it recommends apply specifically to mothers who are HIV seropositive or in high risk groups and their infants. Different management for high and low risk mothers and babies, a two tier approach, presupposes that women at high risk can be identified. With the voluntary antenatal HIV antibody screening currently practised many HIV positive mothers will not be identified antenatally, ${ }^{4}$ and some infants who will subsequently be found to be HIV seropositive will be inappropriately managed as low risk.

Even if universal screening of all women antenatally were introduced, some would be missed because of false negative laboratory tests, women may be tested during the time lag between infection and seroconversion, and some women will refuse to be tested. In addition, as HIV infection becomes more prevalent, it is likely that the currently accepted 'high risk' categories will need to be broadened. In a specialty where emphasis is placed on the normality of pregnancy and in accommodating parents' wishes, it would seem retrogressive to divide all women into high and low risk groups and to modify their management accordingly.

HIV infection is mainly transmitted from mother to infant antenatally; the extent, if any, of transmission at birth and postnatally from breast milk is unclear. The precautions recommended are therefore made primarily for the protection of health workers and not for the baby.

The actual risk of infection to health care staff, through inoculation injuries and after exposure of broken skin and mucous membranes to the blood of infected patients, is extremely low with only 11 cases reported worldwide ${ }^{5}$; of these, only four were infected through inoculation injuries, although hundreds are known to have had penetrating wounds from needles or instruments used for HIV positive patients. There is good evidence, from studies of household contacts of AIDS patients, that HIV is not spread by close social contact nor is there evidence of faecal-oral, airborne, or salivary spread. It is therefore necessary to design infection control policies which, while protecting staff against the small but definite risk of infection, allows the patient to receive full medical care at all times.

Ideally, the same precautions should be applied to all mothers and babies, irrespective of their risk status. This also protects staff from other transmissible viral disorders, particularly hepatitis $B$, which is much more infectious than HIV. This single tier approach has to be balanced against the extra time and inconvenience for staff and expense. Some of the precautions, particularly masks and glasses, make the atmosphere more clinical and less friendly for parents. The acceptance and demand for more stringent precautions is also influenced by the prevalence of HIV in the community, which varies widely in different parts of the country.

\section{Intrapartum care and delivery}

At delivery, large amounts of blood, amniotic fluid, and lochia, all of which are potentially infective, are shed in an uncontrolled way. The RCOG report suggests that from now on, even at normal deliveries of low risk women, a gown should be worn over a plastic apron together with sterile gloves to avoid inadvertent and often unrecognised soiling of clothes. In addition it recommends that boots or 
shoes covered with plastic overshoes should be worn to prevent needle stick injuries to the feet and that all protective clothing and contaminated footwear be changed whenever staff leave the area.

Extra precautions are recommended during labour and delivery of HIV seropositive and high risk women. For intrapartum fetal monitoring, fetal scalp electrodes and fetal blood sampling should be avoided when possible as they result in a small open wound in a contaminated area and place the fetus at increased risk of infection. The delivery should be undertaken only by the minimum number of qualified doctors and midwives. At delivery, because of the variable and sometimes unexpected and explosive shedding of amniotic fluid, blood, and lochia, the report strongly recommends that all staff should wear full protective clothing with a gown over a plastic apron, surgical gloves, cap, mask, eye protection, and protective footwear.

In spite of the difficulties already described in identifying women at high risk and the undesirability of labelling and managing them differently the report considers that a two tier infection control policy is adequate for the United Kingdom at present. A particular problem in adopting a single policy for all women is the recommendation to avoid using fetal scalp electrodes and fetal blood samples. There is clearly an urgent need to develop noninvasive techniques for intrapartum monitoring.

\section{Care of the newborn infant in the labour ward}

When the mother is seropositive or in a high risk group the report advocates that the paediatrician performing neonatal resuscitation should also wear full protective clothing including gloves, a mask, and clear glasses for those not wearing spectacles. If a single tier code of practice is introduced this full protective clothing will be needed whenever resuscitation is performed. In emergencies, the minimum protection of gloves, mask, and eye protection should be used and need to be left readily to hand on the resuscitation table.

For all babies the report recommends that oral mucus extractors should no longer be used but replaced by mechanical suction or bulb syringes. The practice of aspirating meconium directly by oral suction through an endotracheal tube must also clearly be abandoned. This means that endotracheal tubes with a side vent attached directly to mechanical suction on the resuscitation table or a suction catheter passed down inside the endotracheal tube have to be used instead. Alternatively the suction catheter itself can be passed directly between the vocal cords which has the advantage that a wider bore catheter can be used, but is technically more difficult. To aspirate meconium immediately after delivery of the baby's head, mechanical suction apparatus that can only generate low pressures suitable for infants, needs to be used. The bulb syringes mentioned in the report are not currently available in Britain and modified oral mucus extractors are being developed and need to be evaluated. Bag and mask apparatus and laryngoscopes and other non-disposable items used for resuscitation will require sterilisation after use.

\section{Cord blood sampling}

Cord blood sampling is potentially hazardous because of the risk of blood spurting on the attendant and of needle stick injury. Cord sampling is desirable in the infants of seropositive and high risk women for testing for HIV antibody, antigen, and culture, having obtained maternal consent beforehand. The report suggests that instead of bleeding the umbilical cord directly into laboratory tubes a disposable funnel should be used to reduce contamination to the outside of the tubes. Unfortunately this technique is still messy and contamination with maternal blood is likely, making it unsuitable for many investigations. Whenever sampling is performed using a needle and syringe, clearly great care must be taken to avoid needle stick injury.

\section{Washing of newborn infants}

After clamping the cord, if the baby's condition allows, the report recommends that infants born to seropositive and high risk mothers should be washed with soap and water in the delivery room to remove any maternal blood and amniotic fluid. This recommendation is made as HIV virus has been found under experimental conditions to survive even in dried blood. Should this approach be adopted for all babies? Many mothers will doubtless be pleased to have their baby clean and not caked with dry blood for several days. What would be unwelcome would be the return of infants requiring admission to baby units because of hypothermia. So long as this can be avoided, it would be preferable for all babies to be bathed in the labour ward and this has been introduced successfully at St Mary's Hospital recently. Where conditions on the labour ward are unsuitable bathing can be done on admission to the postnatal ward.

\section{Breast feeding}

HIV appears to be present in high titre in cell free breast milk and in the cellular fraction of colostrum of HIV carriers. ${ }^{6}$ This finding does not necessarily 
mean that breast milk is infectious, but there are four case reports where a breast fed infant is thought to have acquired HIV infection postnatally from mothers who received HIV seropositive blood in the postpartum period. ${ }^{7-9}$ However, this may be an unrepresentative group as there may be a higher risk of viral transmission if a woman breast feeds around the time of acquisition of HIV, when there is a peak in antigen concentrations. Some breast fed babies of HIV positive mothers have escaped infection, and it has been suggested that the relative contribution of breast feeding to HIV infection is probably small compared with that of intrauterine transfusion. ${ }^{10} \mathrm{~A}$ recent circular from the Department of Health recommends that to avoid possible added risk to the infant, all mothers known to be HIV infected should be discouraged from breast feeding. ${ }^{11}$ With the evidence currently available, it appears prudent that women who are HIV positive should be counselled about possible risks and not encouraged to breast feed. The circular also extends this advice to include not only mothers known to be HIV infected but to all high risk women. As this includes women who have had sexual relations with people living in African countries (other than those on the Mediterranean) or those who have had sexual partners who have done so, this will apply to a significant proportion of women in many inner city areas. As this recommendation is likely to affect adversely the current advocacy of breast feeding and the vast majority of these women will not be infected it has caused disquiet among many health care professionals. ${ }^{12}{ }^{13}$ Modification of this recommendation is expected in the near future. While these guidelines apply to the United Kingdom, in developing countries where bottle feeding may not be safely achieved, the risk from abandoning breast feeding needs to be weighed carefully against the potential risk of acquiring HIV infection.

Guidelines are also given in the Department of Health's letter regarding human milk banking. As with other donated tissues or organs, mothers wishing to donate milk should first answer a questionnaire to exclude those at high risk and should be known to be HIV antibody negative. The milk must be pasteurised and records maintained about the donors and recipients of the milk. Should a clinician wish to give unpasteurised donated human milk, the mother should be at low risk and antibody negative for HIV, the unpooled milk should be frozen and stored for three months after which the mother should be retested and, if negative, the specimen of milk can be used. The mother should continue to be HIV serotested monthly while she donates milk. The need to adhere to these guidelines is likely to result in an appreciable reduction in human milk banking and has led to the closure of some milk banks.

\section{Postnatal care of the newborn infant}

Once the baby of an HIV seropositive or high risk mother is washed free of blood he can be handled in the normal way, without gloves, and should be cared for in the same room as his mother. The report states, however, that gloves should be worn when performing any procedure involving blood, including blood sampling, administration of intramuscular injections, and when attending to the cord. All blood samples including Guthrie cards should be labelled 'biohazard' and placed in individual plastic bags. Bilirubin samples should not be centrifuged or estimated in a ward laboratory. Gloves should also be worn when passing nasogastric tubes or dealing with vomit or body fluids.

When babies require intensive care, it recommends the wearing of gloves whenever the baby is handled and full protective clothing including visors for invasive procedures.

If paediatricians are going to achieve a uniform approach in caring for all infants irrespective of their HIV status, the most important change in clinical practice surrounds the handling of blood. In contrast with adults, safe blood sampling is difficult in babies as their blood vessels are small and they do not hold still. Whenever a procedure is performed where blood may come into contact with the attendant's skin, surgical or latex gloves should be worn. Surgical gloves are expensive but should be used for invasive procedures as they are currently the only gloves with a British Standard Institution specification. Gloves made of vinyl or copolymer do not provide adequate protection for handling blood. Although many paediatricians dislike wearing gloves for intricate procedures, surgeons regularly perform delicate operations in gloves and reluctance among paediatricians should be overcome. Skin abrasions should be covered by closed unperforated waterproof plaster. Assistance is needed though when fixing cannulas in place as the adhesive tape sticks to the gloves. When arterial cannulation or any invasive procedures are performed, full protective clothing, including eye protection and surgical gloves, should be worn.

In intensive care, the RCOG report advocates that gloves should be worn whenever babies of HIV positive mothers are handled. The basis of this is that these babies have intravenous infusions which often need attention or resiting and are potential sources of blood escape. Should this be extended to cover all babies in intensive care units? Staff prefer not to have to put on gloves repeatedly as it is time 
consuming, handling babies is less pleasant, and gloves are expensive. An alternative is to wear gloves all the time rather than changing repeatedly. Such an approach has already been adopted by some health professionals - for example, dentists, and the gloved hands are washed between patients in the usual way. However, the washing of gloved hands is not recommended as detergents cause the gloves to deteriorate and there may be enhanced penetration of liquids through undetected holes. ${ }^{14} \mathrm{~A}$ further disadvantage is that staff may be tempted to handle babies without prior handwashing, lulled into a false sense of security because they are wearing gloves. In addition, prolonged wearing of gloves is found by some to irritate the skin. Another approach is to advise staff to wear gloves only when they are taking or handling blood or if skin contact with blood is likely. Wearing a fresh pair of gloves for each procedure is clearly more hygienic and it also has the considerable advantage of a single policy for all babies whether in intensive or special care or on the postnatal wards irrespective of their mothers' HIV status. Further information on the advantages and disadvantages of these different approaches is required. Gloves do not provide protection against needle stick injury, and more attention will need to be paid to good technique to reduce this risk. Needles must not be resheathed but placed immediately into safe sharps disposable containers.

A further area of improvement required is in the handling of blood tests in ward laboratories. Clinicians performing these tests need to be trained and equipment maintained so as to conform with accepted laboratory standards of practice. A particular problem lies in safe bilirubin estimation in ward laboratories using blood samples taken into glass micropipettes. Some bilirubinometers use microcuvettes, requiring the glass pipette to be broken to transfer the plasma, a potentially hazardous procedure. Such practices must be modified.

No mention is made in the report of the expense of any of their recommendations. The increased use of protective clothing and gloves and alterations on ward laboratories are all going to involve increased expenditure, and priority should be given for adequate funds.

The field of HIV infection is constantly changing and guidelines will have to be regularly reassessed. The RCOG has already held a workshop on AIDS and obstetrics and gynaecology, the proceedings of which have been published, ${ }^{15}$ and a second edition of the report will be produced. HIV infection has reminded us to respect blood as a potentially infectious fluid. In neonatal paediatrics we should try to avoid a two tier approach, with special policies for infants born to seropositive or high risk mothers. Instead, we must ensure that we provide routine clinical care of sufficiently high standard to be satisfactory for all infants, irrespective of their HIV status.

\section{References}

${ }^{1}$ PHLS Communicable Disease Surveillance Centre, 1988/27.

${ }^{2}$ Hoff R, Berard VP, Weiblen BJ, Mahoney-Trout L, Mitchell M, Grady GF. Seroprevalence of human immunodeficient virus among childbearing women. $N$ Engl $J$ Med 1988;318:525-30.

${ }^{3}$ Report of the RCOG Sub-Committee on problems associated with AIDS in relation to obstetrics and gynaecology. London: Royal College of Obstetricians and Gynaecologists, 1987.

${ }^{4}$ Krasinski K, Borkowsky W, Bebenroth D, Moore T. Failure of voluntary testing for HIV to identify infected parturient women in a high-risk population. $N$ Engl J Med 1988;318:185.

5 Expert Advisory Group on AIDS. AIDS: HIV-infected health care workers. London: HMSO, 1988.

${ }^{6}$ Thirty L, Sprecher-Goldberger S, Jonckheer T. Isolation of AIDS virus from cell-free breast milk of three healthy virus carriers. Lancet 1985;ii:891-2.

7 Ziegler JB, Cooper DA, Johnson RO, Gold J. Postnatal transmission of AIDS-associated retrovirus from mother to infant. Lancet 1985;i:896-7.

${ }^{8}$ Lepage P, Van de Perre P, Carael M, et al. Postnatal transmission of HIV from mother to child. Lancet 1987;ii:400.

9 Weinbreck P, Loustand V, Denis F, Vidal B, Mounier D, De Lumley L. Postnatal transmission of HIV infection. Lancet 1988;i:482.

10 Senturia YD, Ades AE, Peckham CS, Giaquindo C. Breastfeeding and HIV infection. Lancet 1987;ii:400-1.

11 Acheson D, Poole AAB. HIV infection, breastfeeding and human milk banking. London: DHSS, 1988.

12 Logan S, Newell M-L, Ades T, Peckham CS. Breast-feeding and HIV infection. Lancet 1988;i:1346.

13 Brierley J, Roth C, Warwick C. Breast-feeding and HIV infection. Lancet 1988; 1 :1346.

14 Centers for Disease Control. Universal precautions for the prevention of transmission of HIV, HBV and other blood borne pathogens in health care settings. MMWR 1988;37:374-88.

15 Royal College of Obstetricians and Gynaecologists. Aids and obstetrics and gynaecology. Proceedings of the Nineteenth Study Group. London: Royal College of Obstetricians and Gynaecologists, 1988.

T Lissauer

Department of Paediatrics, St Mary's Hospital, London W2 1NY 